\title{
SUGGESTIONS FOR THE MORE READY EMPLOYMENT OF ADAMS' METHOD OF DETERMINING FAT IN MILK.
}

By A. H. Allen and W. Chattaway.

Read at the Meeting, 10th February, 1886.

Tre formal recommendation by the Milk Committee of this Society of the method of determining fat devised by Mr. Adams, has forced the process on the attention of all Analysts, including those who believe the method of fat extraction they have hitherto used to be fairly satisfactory.

It being an admitted fact that most of the methods of determining fat hitherto employed are liable, under certain circumstances, to give results considerably below the truth, chemists are naturally inclined to look favourably on any process by which perfect extraction of the fat from the milk-residue can be ensured. It must further be admitted that the conditions under which the fat is extracted in Adams' method are such as afford a prima facie probability of thorough exhaustion of the residue.

Further, from the analytical data published by the Committee since the date (December 9th, 1885) when they asked the Society to "show its confidence" by then and there accepting their recommendation to adopt $\mathrm{Mr}$. Adams' method as the official process, it appears extremely probable that the procedure in question is theoretically perfect. While preferring to reserve a positive opinion on this point until we have had a longer and more rigid experience of the method, it appears to us that the process, as prescribed by the Milk Committee, is not sufficiently practical to render its general adoption by the members of the Society probable, or even possible, having regard to the circumstances under which many of their analyses require to be made. The chief objections which have suggested themselves to us, and which were pointed out by one of us to the meeting on December 9th, are:-(1) That, if the instructions of the Milk Committee be followed, two weighings are necessarily made of each sample of milk, even though other data may subsequently show that the determination of fat is not requisite. (2.) That these weighings must be made very promptly, or the milk will have turned sour, and can no longer be fairly absorbed by the paper. (3.) That it is often practically impossible to make these weighings before the milk has commenced to change.

To the first of these objections, Mr. Adams replies that, practically, the whole of the 5 c.c. measured into the beaker is soaked up by the paper coil, and hence the two weighings may be dispensed with. As for sour mill, Mr. Adams recommends the use 
of ammonia, which, he states, will restore the most curdled milk to the state of emulsion. The Committee, in the official process they recommend, make no reference to measuring, instead of weighing, the milk; and do not suggest the use of ammonia. Hence, the presumption is that they insist on the double weighing, and either object to the use of ammonia on chemical grounds or have never realised the occasion for prescribing a process for analysing slightly sour milk.

While reserving our opinion as to the accuracy and value of Adams' method of treating milk for the extraction of the fat, we have felt the absolute necessity of some simplification of the manipulation if the process is to be generally adopted; and, as the outcome of several experiments on the mechanical details of the process, we have devised the following plan of making the paper coils, which plan we venture to think an improvement of the original method:-

We roll up with the paper a piece of string previously boiled with water containing a little sodium carbonate (in order to remove size, \&c.), which serves to keep the concentric folds of the coil from contact with each other. We also tie on to the lower end of the coil a piece of filter-paper about $3 \frac{1}{2}$ inches in diameter. This is made of thinner paper than the coil itself, and is pleated round the sides of the coil, somewhat after the manner in which a dispenser folds the paper cap over the cork of a bottle of medicine. This device provides the coil with a bottom, and enables us to deliver 5 c.c. of milk as fast as it will run from the measuring pipette on to the upper end of the coil, which should, meanwhile, be suspended from a suitable support. In this manner the necessity of weighing of the milk absorbed by the coil is wholly avoided.

As the novelty attaching to our mode of operating really lies in the construction of the paper coil, it is desirable to describe the method of making it in more detail.

The strip of paper we employ is of the same dimensions and quality as that used by $\mathrm{Mr}$. Adams ; but each concentric ring of paper in the coil is kept equi-distant from the next by means of a piece of string, which is threaded down either side of the

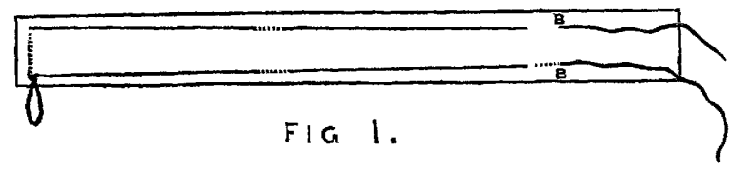
paper, about half-an-inch from the edge, as shown in the accompanying sketch (Fig. 1). It will be seen that there is a loop, formed by knotting the doubled string, which loop protrudes from the centre of the coil when finished, and is useful for hanging it up while being charged with milk, and during the subsequent process of drying.

A small glass rod (or other suitable article) is used to roll the paper upon, the rolling being commenced at the loop-end of the strip. When the coil has been rolled as far as the last holes in the paper (в.в.), the two ends of the string are tied in a knot as close to the paper as possible. A hole is then made through the centre of the paper, immediately under the knot, and of sufficient size to allow the knot to go through. The winding of the remaining three inches of the paper is then completed, and a pleated cap of filtering paper placed over the bottom of the coil. A hole is made through this cap at the side, just where the knot occurs. The ends of the string are 
threaded through this hole, and the cap and coil are then secured by merely tying the two ends of the string round the cap. The finished coil has a diameter of $\frac{7}{8}$ inch, and can then be extracted with ether, in the usual way, to remove traces of resin, \&c.

The advantages we claim for the coils made in the foregoing manner, specimens of which we produce, are as follows:-

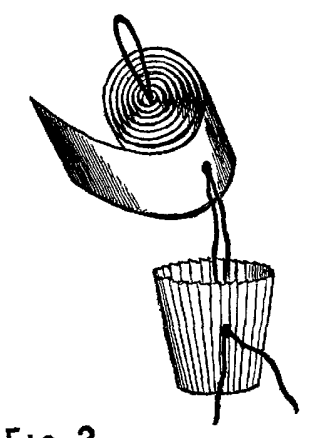

Fig 2 .

The concentric folds of paper being kept separate by the string, the full extent of surface is exposed, and the process of drying is correspondingly facilitated.

The cap of filter-paper at the bottom, aided by the obstruction produced by the string, renders it possible to pour the milk at once on to the open end of the coil while it is suspended by the loop of string. No loss by filtration of the milk through the cap has ever occurred to us. This fact enables us to dispense with the two weighings which militate so powerfully against the general adoption of the original mode of operation. With the modified coil, 5 c.c. of the milk can be taken up in a pipette, and at once run on to the suspended coil. The density having been previously observer, the weight employed is, of course, accurately known.

Although in general, and when operating on fresh milk, we consider the use of a definite measure of the sample has marked advantages, the use of a known weight of milk is equally simple. The amount to be taken can be weighed in a tared tube or small beaker, poured on to the coil, and the vessel rinsed out with a few drops of water, which in their turn are added to the coil. The last plan is equally available for sour milk, a weighed quantity of which may be at once poured on the centre of the coil-the modified plan having a marked advantage in this respect over the coil as originally constructed. If it be considered desirable to employ ammonia, its addition to the weighed quantity of milk, before pouring on to the coil, presents no difficulty.

A number of coils being charged with milk, they can be conveniently hung on rows of pins in a vertical Stone's box, or similar receptacle, and can be kept there for an indefinite period.

Conclúsion of the Society's Proceedings. 